\title{
Primary bradycardia and the evoked cardiac response in the OR context
}

\author{
ROBERT J. BARRY \\ University of New South Wales, Kensington, N.S.W., Australia
}

\begin{abstract}
This study sought evidence for linkages between the deceleratory evoked cardiac response (ECR) commonly observed in the OR context and the primary bradycardia of the Laceys. Individual HR protocols from a parametric study of the unitary OR were examined for both cardiac effects. Because of the small amount of data, a procedure of time-series analysis was initially used to remove respiratory sinus arrhythmia noise from the individual protocols. Previous criticism of the uncritical use of this procedure in situations with systematic respiratory changes led to statistical adjustment for this variable by covariance analysis. Evidence was obtained for a brief deceleratory ECR which failed to show the effects of stimulus novelty or intensity that were apparent in the GSR. This slowing of the heart rate began in the cardiac cycle containing the stimulus onset, as expected of primary bradycardia. The magnitude of this immediate slowing showed the dependence upon cardiac phase associated with primary bradycardia, and was also unaffected by stimulus novelty or intensity. These results support a view of the ECR as a cumulative primary bradycardia reflecting stimulus registration, a preliminary process in OR elicitation.
\end{abstract}

The use of heart rate changes to index the orienting response (OR) followed from the influential review paper of Graham and Clifton (1966). E. N. Sokolov, whose writings popularized the OR in Western psychology, rarely referred to heart rate changes, and then only in his early works: Sokolov $(1960,1963)$ apparently considered heart rate acceleration as a measure of the OR. In contrast, Graham and Clifton reviewed the available literature and concluded that cardiac deceleration satisfied the requirements of an OR index. Their equating of cardiac deceleration with the OR appears to have become the received, traditional view of the functional nature of cardiac deceleration, in spite of some evidence to the contrary. The main empirical argument against this contention of Graham and Clifton's comes from a number of studies (e.g., Barry, 1977a, 1977b; Hulstijn, 1978; van Olst, Heemstra, \& ten Kortenaar, 1979) which indicate that the evoked cardiac response (ECR) of heart rate deceleration produced by simple stimulation fails to habituate with stimulus repetition. Such a failure is important, because habituation is an essential characteristic of orienting behavior and a necessary property of any physiological measure purporting to index that behavior.

\footnotetext{
A preliminary version of this paper was presented at the 22nd Annual Meeting of the Society for Psychophysiological Research in Minneapolis, Minnesota, in October 1982. My thanks are due to Tim Lobstein for supplying the program CHANT, which was used for time-series analysis of heart rate. Address reprint requests to Robert J. Barry, School of Education, University of New South Wales, Kensington, N.S.W. 2033, Australia.
}

If the ECR of heart rate deceleration is not an index of the OR, we require an alternative conceptualization that can accommodate its established characteristics. Barry $(1981,1982$, in press) has recently presented a new theory of preliminary processes in OR elicitation which is compatible with a nonhabituating ECR. This theory was developed in response to the observed fractionation of phasic responses in the OR context (Barry, 1977a, 1977b, 1978b, 1979b), a fractionation incompatible with Sokolov's unitary OR concept. In this new theory, the ECR is hypothesized to reflect the process of stimulus registration. This hypothesis was based upon the observed independence of ECR magnitude from stimulus novelty and magnitude: similar ECRs appear to be elicited by any OR-compatible stimulus. Such a relationship with the stimulus qua stimulus is suggestive of an early stage in stimulus processing prior to the coding of stimulus parametersthat is, the initial process of stimulus registration.

About the time that work on the unitary OR and this hypothetical process of stimulus registration began to appear from this laboratory some 5 years ago, the Laceys began to publish their work on what they later called "primary bradycardia" (Lacey \& Lacey, $1977,1978,1980$ ). They observed that brief auditory and visual stimulation produced an increase in pulse duration in the very cardiac cycle that contained the stimulus onset, as well as in the subsequent cycle. This increase in period was dependent upon when stimulation occurred in the cardiac cycle. The earlier in the cycle that stimulation occurred, the greater was the increase in the duration of that cycle. The later 
in the cardiac cycle that stimulation occurred, the less was the extension of that cycle, but the greater was the effect upon the subsequent cycle. The rapid nature of such a response precludes such variables as baroreflex mediation, leading Lacey and Lacey (1980) to note parallels with the primary bradycardia found with stimulation of the lateral hypothalamus in animal studies. Kaufman, Hamilton, Wallach, Petrik, and Schneiderman (1979) had reported such a response in rabbit without the marked blood pressure changes associated with baroreflex mediation. This response was abolished by bilateral sectioning of the vagus nerves. Hence, the Laceys considered their human cardiac response as a vagally mediated primary (or reflex) bradycardia, which they described as "an index of an early process of stimulus-registration by the nervous system"' (1980, p. 22).

This should be compatible with the stimulus registration process in the Barry model of preliminary processes in OR elicitation, but the relationship between the brief within-cycle bradycardia of the Laceys and the ECR in the Barry model has not been explored. The deceleratory ECR apparently commences in the cycle containing the stimulus onset, reaches a maximum about two beats later, and is followed by a gradual return to baseline (see, for example, Barry, 1977a). This ECR in the OR context is of longer duration and larger magnitude than the primary bradycardia of the Laceys. However, the Laceys have used stimuli of much shorter duration than is common in OR work-typically $100 \mathrm{msec}$ compared with the 2 sec usually employed in our laboratory. Such a difference in stimulus duration could be expected to affect both response magnitude and duration, particularly since temporal summation in primary bradycardia has been demonstrated in animal studies (Evans, 1976; Kaufman et al., 1979). One further fact supports a connection between these two response extremes in humans: Lacey and Lacey (1980) reported that primary bradycardia fails to habituate with stimulus repetition, compatible with our results for the ECR. This suggests that the ECR in the OR context may be a cumulative primary bradycardia reflecting the process of stimulus registration hypothesized in the Barry model. The present study was carried out to investigate this suggestion by exploring the evidence for primary bradycardia in a relatively standard $O R$ paradigm.

A recurrent problem in the measurement of stimulus-evoked cardiac activity is correction for the systematic variation of heart rate with respiration. This effect, known as respiratory sinus arrhythmia (RSA), is observed as an increase in heart rate accompanying inspiration, and a decrease during expiration. In adults, RSA produces a fluctuation of about $5 \%$ of the resting heart rate, with considerable individual variation. Unfortunately, this background noise level is often comparable with the ECR, es- pecially with the innoccuous stimuli common in the OR field (see, for example, Barry, 1978a). A recent approach to this problem has been to use time-series analysis (e.g., Lobstein, 1978; Lobstein, Webb, \& Cort, 1978) to extrapolate prestimulus RSA into the poststimulus period. The ECR is then defined in terms of deviations from these expected values. Barry (1979a) has suggested that the use of such techniques without consideration of respiratory changes may lead to invalid conclusions: a computer simulation of HR protocols across a change in respiration indicated that spurious ECRs could be generated. This is of importance, since many of the studies from this laboratory have reported reliable increases in respiratory period at stimulus onset and systematic reductions in the magnitude of this respiratory pause with stimulus repetition. Although the spurious ECRs might be rather small, the habituation of respiratory pause could produce systematic changes which would appear to indicate habituation of the ECR with stimulus repetition. While the noise introduced by these spurious ECRs is markedly less than that of RSA itself, any systematic effects over trials require correction, particularly in the context of the dispute over habituation of the ECR alluded to above. While Barry (1979a) suggested that the entire problem would be eliminated if HR was scored on a timebase defined by respiratory activity, the systematic trials effect could be corrected by covariance analysis with respiratory pause as the covariate for each trial's derived ECR.

Thus, the present study used time-series analysis, corrected for spurious systematic trial effects, to generate estimates of both primary bradycardia (in the first poststimulus cardiac cycle) and traditional ECRs (in the first five poststimulus beats) in individual protocols from a fairly standard OR experiment. Examination of the covariation of these measures over stimulus intensity and novelty is the first step in testing the hypothesis that the ECR in the OR context is a cumulative primary bradycardia.

\section{METHOD}

Twenty-four male university students, under 25 years of age, each received eight cycles of $2-\sec 1000-\mathrm{Hz}$ tones at $20,30,40$, and $50 \mathrm{~dB}$ SPL via circumaural headphones. The order of tone intensities was varied over subjects, with each subject receiving a different one of the 4 ! possible stimulus-intensity cycles. Mean interstimulus interval was $60 \mathrm{sec}$. The subjects were instructed to "pay close attention" to the stimuli and were warned that, in order to be included in the study, they would have to convince the experimenter, at a postexperimental quiz, that they had "paid attention to the tone series." This ensured that the stimuli were of some significance. The experiment has been reported in more detail in Barry (1977a). EKG from lead 1 was recorded on a Grass IIID polygraph with a paper speed of $15 \mathrm{~mm} / \mathrm{sec}$. Respiratory activity was recorded, from an E \& $M$ bellows pneumograph around the subject's chest, on an E \& M Physiograph Six with a paper speed of $2.5 \mathrm{~mm} / \mathrm{sec}$. GSRs, measured as ac-coupled skin resistance responses (SRRs), were also recorded on this de- 
vice. Other physiological measures were recorded on each chart recorder, but are not relevant to the present report.

For the purpose of the present study, EKG protocols were examined to identify stimuli that occurred early or late in the cardiac cycle. Stimuli which could not be unequivocally so identified, because of their coincidence with the $\mathbf{R}$ wave or the midpoint of the cycle, were omitted. For each subject, the first and last stimuli, at both 20 and $50 \mathrm{~dB}$, which occurred early and late in the cardiac cycle, were identified. For each of these eight stimulus presentations, the HR of 10 prestimulus and 10 poststimulus cardiac intervals (the first of the poststimulus cycles contained the stimulus onset) were scored. These $20 \mathrm{HR}$ values were separately submitted to CHANT, the program for time-series analysis of HR developed by Lobstein (1978). Deviations from extrapolated HRs were considered as the ECR, and such deviations in the first poststimulus cycle were taken as reflecting primary bradycardia. In order to allow correction for respiratory effects the value of respiratory pause for each relevant stimulus was obtained from Barry (1977a), where respiratory pause was defined as the percentage increase in the duration of the respiratory cycle containing the stimulus onset relative to the prestimulus cycle. The GSR was also extracted for each relevant stimulus and examined for the effects of the stimulus variations of interest here.

\section{RESULTS}

\section{GSR}

The left panel of Figure 1 indicates mean GSRs as functions of stimulus intensity ( $20 \mathrm{vs.} 50 \mathrm{~dB})$, trial (first vs. last), and cardiac phase (early vs. late). A large number of zero responses precluded parametric analysis of this OR measure. Accordingly, separate Wilcoxon matched-pairs signed-ranks tests were conducted for the GSRs over each independent variable. There was a significant ${ }^{1}$ intensity effect $(Z=3.41$, $\mathrm{df}=23$ ), with the $50-\mathrm{dB}$ stimuli eliciting larger responses than the 20-dB tones. Habituation was indicated by the significant trials effect $(Z=3.32$, $\mathrm{df}=23$ ). The effect of cardiac phase, although small, approached significance $(\mathrm{Z}=1.90, \mathrm{df}=23, \mathrm{p}=.06)$.

\section{Respiratory Pause}

The changes in respiration at each stimulus presentation were subjected to a three-way repeated- measures ANOVA which examined the effects of stimulus intensity, trial, and cardiac phase. The effects of these variables are shown in the right panel of Figure 1. The expected trials effect in respiratory pause, although evident in Figure 1, failed to reach statistical significance $[\mathrm{F}(1,23)=2.673, \mathrm{MSe}=$ 287.327]. As is apparent from the figure, there was little suggestion of intensity or cardiac phase effects in respiratory pause, with all Fs less than unity. No evidence for interactions between these variables was apparent.

\section{ECR}

The mean beat-by-beat raw HR is shown on the left in Figure 2 for each of the eight stimulus types. Each curve is the mean over 24 subjects for one combination of stimulus intensity, trial value, and cardiac phase at stimulus onset. To the left of each curve is shown the mean HR of the immediately prestimulus cardiac cycle, which is the first point in each curve (labeled beat 0 ). To the right of each curve, the standard error of the HR of poststimulus beat 10 is shown as an indication of cardiac variability. These raw HR curves indicate that a deceleratory response occurred following stimulus onset, as expected from previous work. The individual response curves underlying these were submitted to CHANT, producing eight new curves for each subject. Each of these curves represents the departure of the observed HR from that extrapolated from the prestimulus period, and may be taken as a beat-by-beat ECR corrected for RSA. These data, on the basis of both previous work and the raw response curves discussed above, were analyzed in two parts: over the first five poststimulus beats and then over the second five poststimulus beats. The first of these ranges corresponds to the common limits used to define the ECR in OR studies (e.g., Barry, 1977b; Jackson, 1974), and the second covers the recovery limb following this response. For each of these ranges, a four-way repeated measures analysis of covariance, with the
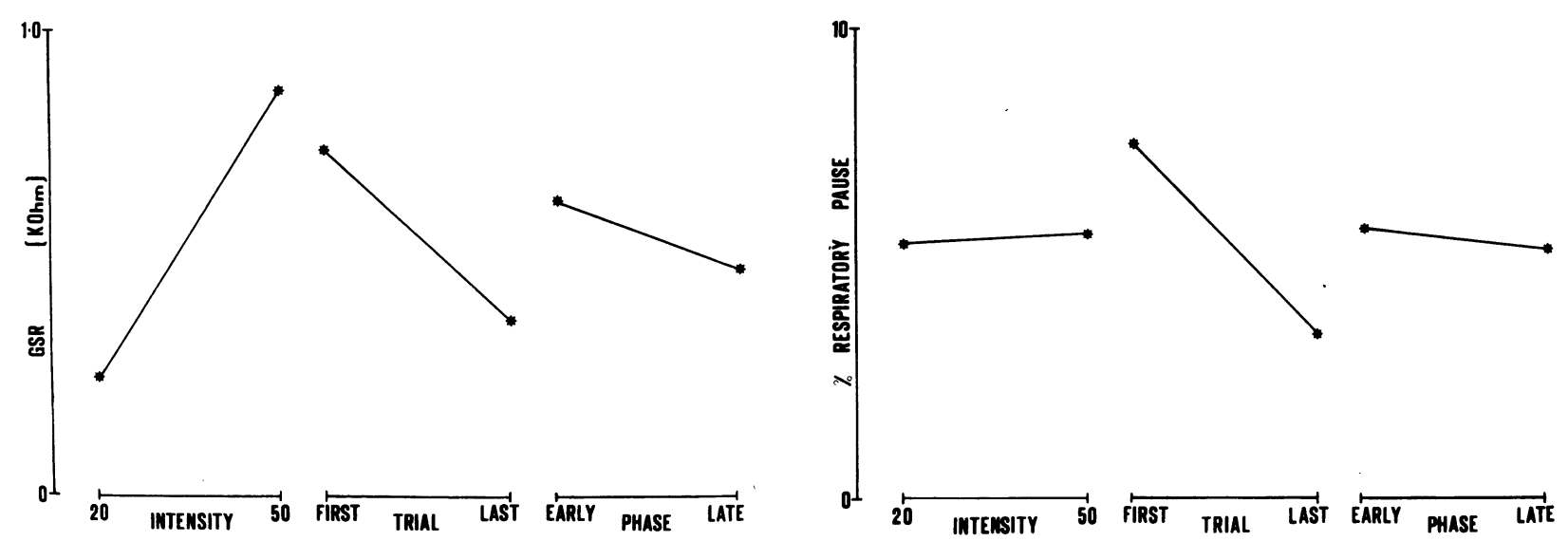

Figure 1. The left panel shows mean GSRs (measured as SRRs in $\mathrm{K}$ ohms), and the right panel shows respiratory pause (percentage increase in period), as functions of stimulus intensity ( $20 \mathrm{vs.} 50 \mathrm{~dB}$ ), trial (first vs. last), and cardiac phase (early vs. late). 


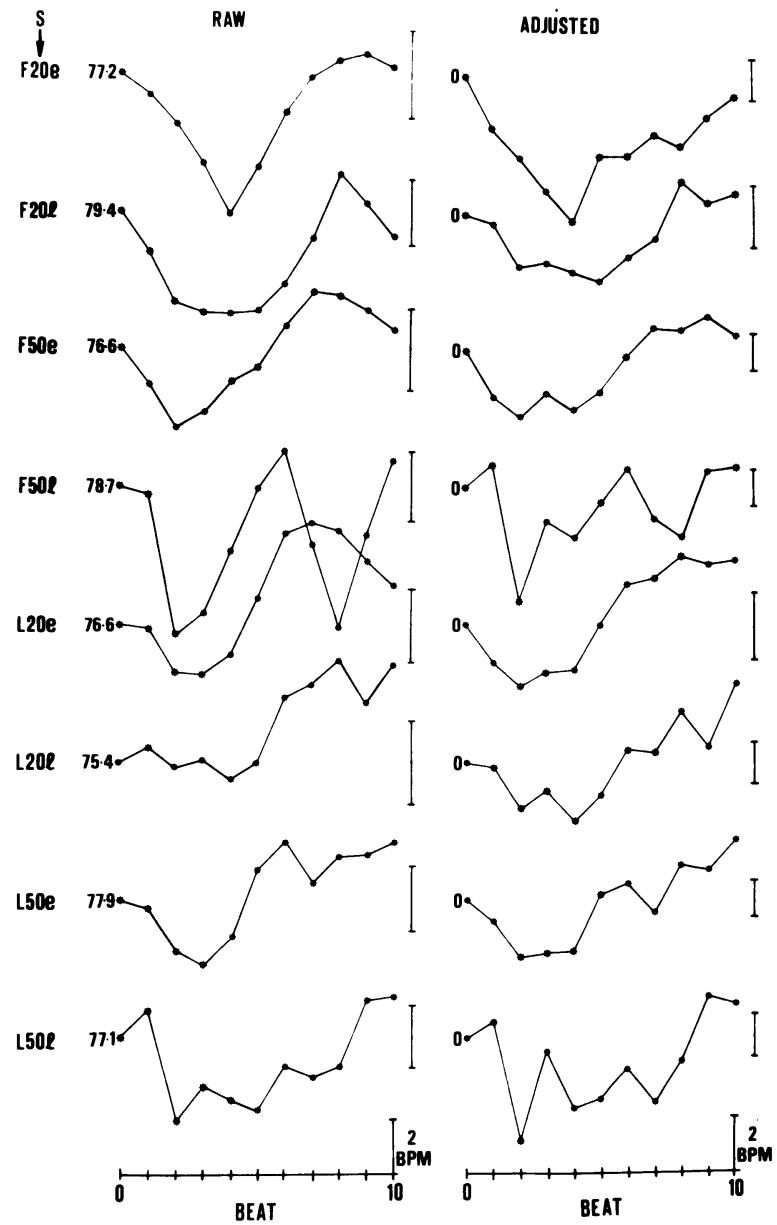

Figure 2. Mean HR curves over subjects for each of the eight stimulus types. The stimulus type (S) is indicated on the extreme left of the figure in terms of trial (first vs. last), stimulus intensity (20 vs. $50 \mathrm{~dB}$ ), and cardiac phase (early vs. late). Raw HR curves are displayed on the left, and adjusted deviations from predicted HR are on the right. Immediately to the left of each curve is the numerical value of the prestimulus mean (Beat 0 ) in bpm. The vertical bar to the right of each curve represents the standard error of the last point in the curve.

associated respiratory pause measure as the covariate, was carried out. Three of the factors involved (trials, intensity, and cardiac phase) were the same as in the ANOVA used with respiratory pause above. The other factor, beats, was analyzed for linear and quadratic trends only. The adjusted means from these analyses are shown on the right-hand side of Figure 2, together with the standard errors (unadjusted) of the Beat 10 mean. Some smoothing of the curves, together with a reduction in the variability indicated by the standard errors, is readily apparent.

The average of these adjusted curves is shown in Figure 3, which represents a mean RSA-corrected ECR adjusted for habituation in the respiration changes at stimulus onset. The analysis indicated that the mean of the first five beats was significantly different from zero $[F(1,22)=8.037, \mathrm{MSe}=121.134]$, confirming that the cardiac deceleration produced by stimulation was statistically significant. There was a significant quadratic trend over these beats $[F(1,23)$ $=14.064, \mathrm{MSe}=21.007$ ], confirming the peaking of the ECR in the first five poststimulus beats. No other main effects or interactions approached significance. The analysis over Poststimulus Beats 6 to 10 indicated a significant trials effect $[F(1,22)=5.760$, $\mathrm{MSe}=74.509$ ], shown by the broken lines in Figure 3. This suggests a greater rebound effect following the deceleratory ECR of the later trials. This rebound effect was represented by a significant linear trend over beats $[\mathrm{F}(1,23)=4.940, \mathrm{MSe}=75.353]$. No other main effect approached significance. The three-way interaction between linear trend over beats, trials, and intensity, approached significance $[\mathrm{F}(1,23)=4.088, \mathrm{MSe}=13.412, \mathrm{p}=.055]$. Inspection of Figure 2 (right-hand side) suggests that this reflects the faster recovery with the 50-dB stimuli of the later trials. There was also a significant four-way interaction between quadratic trend over beats, trials, stimulus intensity, and cardiac phase $[F(1,23)=$ $7.254, \mathrm{MSe}=10.037$ ]. This is virtually uninterpretable, and will not be discussed further. No other interactions approached significance.

The largest deceleration in the first five poststimulus beats was taken as a measure of the ECR for each trial. These data were analyzed with a threeway repeated-measures analysis of covariance, analogous to the ANOVA used in the analysis of respiratory pause but with respiratory pause as the covariate. Although the mean ECR was significantly greater than zero $[\mathrm{F}(1,22)=73.687, \mathrm{MSe}=48.517]$, none of the main effects or interactions approached significance. This lack of sensitivity in the ECR is apparent in the left panel of Figure 4. It is interesting to note that the trial effect was reduced by the inclu-

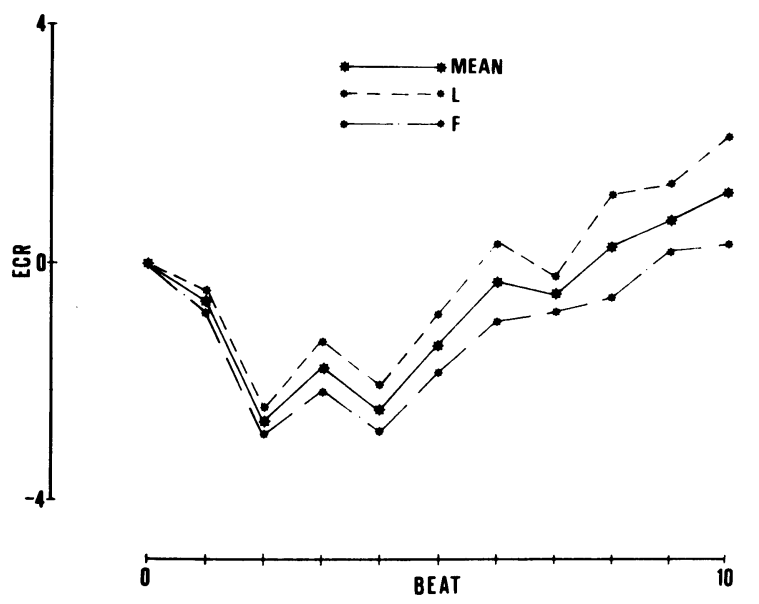

Figure 3. Mean adjusted beat-by-beat ECR (in bpm) over subjects and stimulus types. Dashed lines show the effect of trials (first vs. last). 

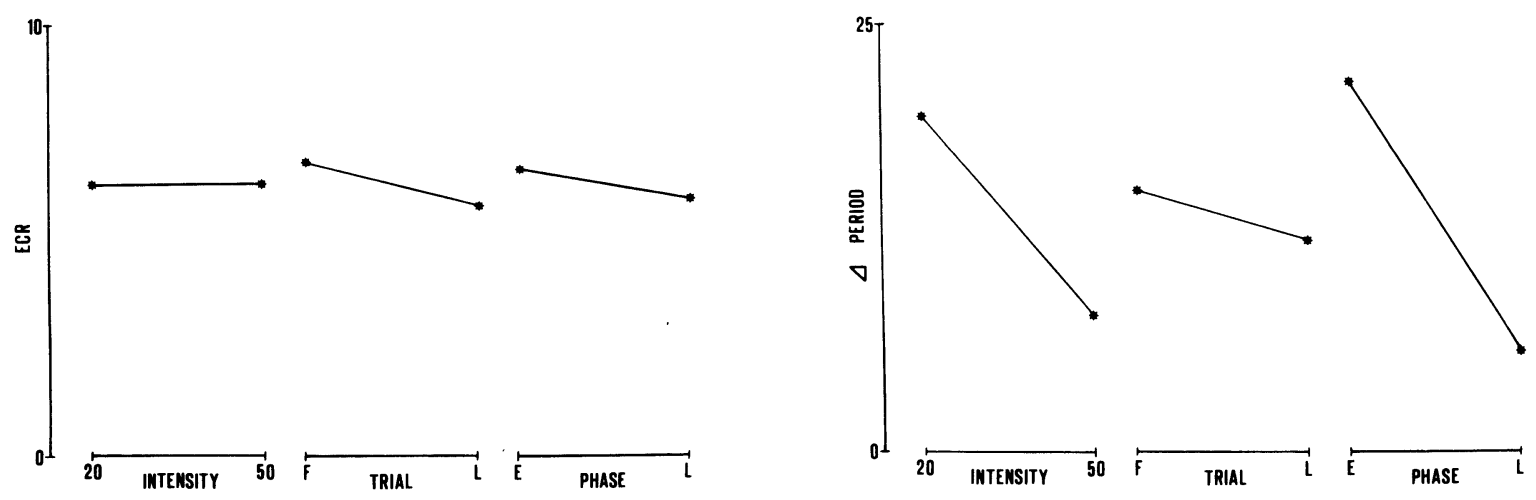

Figure 4. Magnitude of the derived ECR (in bpm) is shown on the left, and change in period (in milliseconds) of the cardiac cycle containing the stimulus onset is shown on the right. Each variable is displayed as a function of stimulus intensity (20 vs. $50 \mathrm{~dB}$ ), trial (first vs. last), and cardiac phase (early vs. late).

sion of the covariate, although this reduction was not statistically significant: the relevant $F$ was reduced to 1.674 in the analysis of covariance described above, compared with 3.024 in the corresponding ANOVA. This latter value suggests that a spurious habituation of the ECR may indeed be obtained by a noncritical application of time-series analysis in these circumstances.

\section{Primary Bradycardia}

The increase in the duration of the cardiac cycle containing the stimulus onset was expressed in milliseconds and subjected to the analysis of covariance described above. The main effects examined are shown in the panel on the right in Figure 4. The effect of cardiac phase was significant $[F(1,22)=4.631$, $\mathrm{MSe}=2541.815$ ], with stimuli occurring early in the cardiac cycle producing a greater slowing of HR than stimuli occurring later in the cycle. Although there is a suggestion of an intensity effect in the figure, this failed to approach significance $[\mathrm{F}(1,22)=1.476$, $\mathrm{MSe}=4413.153]$, and no other effect or interaction approached significance.

In order to estimate the common variance between ECR and the first-cycle primary bradycardia, the correlation coefficient between these measures was calculated for each of the eight stimulus types. This ranged from .02 to .64 , with a mean of .45 . Although significantly different from zero, this indicates that the two measures share only about $20 \%$ of their total variance.

\section{DISCUSSION}

The results obtained with the GSR indicate that the subset of data from Barry (1977a) examined here was based upon sufficient stimulus variation to allow intensity and novelty effects to appear in the OR. In contrast, the deceleratory ECR failed to show either of these effects in the present situation. This is visually apparent from the raw HR protocols shown in Figure 2, and was statistically confirmed for the RSA-corrected ECRs obtained from timeseries analysis. Although acceptance of the null hypothesis does not constitute proof, this result adds to the evidence mitigating against the use of the deceleratory ECR as an index of the OR. This is because habituation with reductions in stimulus novelty, and sensitivity to stimulus magnitude, are defining characteristics of the OR and, necessarily, of its indices. ${ }^{2}$ Thus, while the ECR appears to be part of the complex physiological changes associated with elicitation of the OR, it cannot be used to index the OR. Rather, the present results are compatible with the concept of the ECR as an index reflecting stimulus registration, a preliminary process in OR elicitation. The RSA-corrected ECR, adjusted for systematic respiratory effects, is compatible with previous HR curves obtained in this laboratory, and confirms the validity of response criteria used in previous reports. Thus, the definition of the ECR as the largest deceleration in the first five poststimulus beats appears to be adequate for stimuli with durations similar to the $2 \mathrm{sec}$ commonly used in this laboratory. However, if the ECR is a cumulative primary bradycardia, exhibiting temporal summation, these response criteria may require modification with changes in stimulus duration. Systematic investigation of this question would appear to be warranted by the present results.

The auditory stimuli used in the present study have been shown to produce a prolongation of the cardiac cycle in which the stimulus onset occurs. This immediate effect, which was greater for stimuli occurring early, rather than late, in the cardiac cycle, appears to be identical with the primary bradycardia of the Laceys. It also failed to exhibit significant stimulus intensity or novelty effects, in parallel with the ECR. These findings support the view of the ECR as a cumulative primary bradycardia reflecting an initial 
stage in the processing of the stimulus. Some difficulties with this identification remain. The most obvious of these concerns is the relatively small correlation between the ECR and the primary bradycardia. However, this may be attributable to the fact that the measure of primary bradycardia spanned only the first poststimulus cardiac interval, whereas the ECR measure was taken up to the fifth poststimulus cycle. The present study, with only eight data points per subject, could not examine individual differences in the temporal relationships between these two measures, but such differences could be expected to contribute to the low correlation obtained. In addition, differences in heart rate between subjects would add to the measurement noise obscuring the exact relationship between these measures. It seems reasonable to expect that adequate control of these sources of variation between and within subjects will be reflected in an increase in the apparent strength of the relationship between primary bradycardia and the ECR. Thus, the present study should be considered as a first step in establishing the linkage between the primary bradycardia of the Laceys and the ECR in the OR context.

Finally, this study has demonstrated the usefulness of time-series analysis as a means of removing the effects of RSA from HR protocols. By enhancing the signal to noise ratio, relatively small amounts of data may then yield useful information. However, it would appear that the caution recommended by Barry (1979a), when applying time-series analysis in situations in which systematic changes in respiratory activity are expected, is warranted. The procedure used in this study to correct for such biases in the output of the time-series analysis was statistical, employing analysis of covariance with the respiratory changes as the covariate. The success of such correctional procedures may encourage a wider use of time-series analysis in this area.

\section{REFERENCES}

BARRY, R. J. The effect of "significance" upon indices of Sokolov's orienting response: A new conceptualization to replace the OR. Physiological Psychology, 1977, 5, 209-214. (a)

BARRY, R. J. Failure to find evidence of the unitary OR concept with indifferent low-intensity auditory stimuli. Physiological Psychology, 1977, 5, 89-96. (b)

BARRY, R. J. Disruption of sinus arrhythmia and its relation to the classification of non-habituating OR measures. Physiology \& Behavior, 1978, 21, 25-27. (a)

BARry, R. J. Physiological changes in a reaction-time task: Further problems with Sokolov's dimension of stimulus "significance." Physiological Psychology, 1978, 6, 438-444. (b)

BARRY, R. J. Correction for sinus arrythmia in the evoked cardiac response: A timebase problem. Biological Psychology, 1979, 9, 215-220. (a)

BARRY, R. J. A factor-analytic examination of the unitary OR concept. Biological Psychology, 1979, 8, 161-178. (b)
BARRY, R. J. Signal value and preliminary processes in OR elicitation. Pavlovian Journal of Biological Science, 1981, 16, 144-150.

BARRY, R. J. Novelty and significance effects in the fractionation of phasic OR measures: A synthesis with traditional OR theory. Psychophysiology, 1982, 19, 28-35.

BARry, R. J. Preliminary processes in OR elicitation. Acto Psychologica, in press.

Evans, M. H. Stimulation of the rabbit hypothalamus: Caudal projections to respiratory and cardiovascular centres. Journal of Physiology (London), 1976, 260, 205-222.

Graham, F. K., \& Clifton, R. K. Heart-rate change as a component of the orienting response. Psychological Bulletin, $1966,65,305-320$.

HulstiJn, W. Habituation of the orienting response as a function of arousal induced by three different tasks. Biological Psychology, 1978, 7, 109-124.

JaCkson, J. C. Amplitude and habituation of the orienting reflex as a function of stimulus intensity. Psychophysiology, 1974, 11, 647-659.

Kaufman, M. P., Hamilton, R. B., Wallach, J. H., Petrik, G. K., \& Schneiderman, N. Lateral subthalamic area as mediator of bradycardia response in rabbits. American Journal of Physiology, 1979, 236, H471-H479.

LACEY, B. C., \& LACEY, J. I. Change in heart period: A function of sensorimotor event timing within the cardiac cycle. Physiological Psychology, 1977, 5, 383-393.

LACEY, B. C., \& LACEY, J. I. Two-way communication between the heart and the brain: Significance of time within the cardiac cycle. American Psychologist, 1978, 33, 99-113.

LACEY, B. C., \& LACEY, J. I. Cognitive modulation of timedependent primary bradycardia. Psychophysiology, 1980, 17, 209-221.

LoBste in, T. Detection of transient responses in adult heart rate. Psychophysiology, 1978, 15, 380-381.

Lobste in, T., WebB, B., \& CoRT, J. Background noise levels and heart rate orienting: Response detection using time series analysis. Psychophysiology, 1978, 15, 316-319.

van Olst, E. H., Heemstra, M. L., \& ten Kortenaar, T. Stimulus significance and the orienting reaction. In $H$. D. Kimmel, E. H. van Olst, and J. F. Orlebeke (Eds.), The orienting reflex in humans. Hillsdale, N.J: Erlbaum, 1979.

Sokolov, E. N. Neuronal models and the orienting reflex. In M. A. Brazier (Ed.), The central nervous system and behavior. New York: Macey, 1960.

Sokolov, E. N. Perception and the conditioned reflex. Oxford: Pergamon Press, 1963.

\section{NOTES}

1. Significance level used is $\mathrm{p}<.05$

2. It should be noted that there was a significant trial effect and a trial $x$ intensity interaction apparent in the ECR obtained here, but that these were restricted to Beats 6-10. These late rebound effects are not compatible with predictions from Graham and Clifton (1966) because they were not found in the decelerative phase of the ECR. Furthermore, the interaction effect obtained (in which more intense stimuli produced greater rebound on later trials) would be predicted by OR theory only if the accelerative rebound effect was considered as an OR indicator. Such a proposition has no currency in modern OR literature. The present results thus provide no support for Graham and Clifton's view of the ECR as an $O R$ index.

(Manuscript received October 6, 1982; revision accepted for publication April 11, 1983.) 